\title{
Los actuales retos de la cooperación militar y las capacidades de la Unión Europea*
}

\author{
Current challenges to EU military cooperation and capabilities \\ Francisco José Ruiz González \\ Profesor de la Escuela Superior de las Fuerzas Armadas
}

Sumario: I. Introducción: hitos previos al nacimiento de la PESD.II. El «Objetivo Principal de Helsinki» y la relación con la OTAN.III. Avances de la PESD tras la aprobación de la Estrategia Europea de Seguridad.-IV. La entrada en vigor del Tratado de Lisboa: expectativas defraudadas. $-V$. Ultimas reflexiones y perspectivas de futuro.

Resumen: La Política Común de Seguridad y Defensa de la Unión Europea está lejos de alcanzar el nivel de desarrollo previsto por el Tratado de Lisboa, en especial en lo relativo a la cooperación militar y las capacidades de defensa. En el futuro se deben optimizar las capacidades existentes, desarrollar otras nuevas, avanzar en la especialización de funciones de los Estados miembros, e integrar los diversos programas, todo ello bajo la guía política del Consejo Europeo.

Palabras clave: Unión Europea, PESC, PESD/PCSD, operaciones, capacidades.

Abstract: The Common Security and Defence Policy of the European Union is far from reaching the development level envisaged by the Lisbon Treaty, especially in terms of military cooperation and defense capabilities. In the future, the existing capabilities must be optimized and new ones developed, the specialization of functions of the member states must go on, and the different programs should be integrated, everything under the political guidance of the European Council.

Keywords: European Union, CFSP, ESDP/CSDP, operations, capabilities.

\section{Introducción: hitos previos al nacimiento de la PESD}

Como se afirma en un reciente informe ${ }^{1}$, la Política Común de Seguridad y Defensa (en adelante PCSD) es el eslabón más débil en el proyecto

* Recibido el 16 de marzo de 2015, aceptado el 21 de mayo de 2015.

${ }^{1}$ More Union in European Defence, Centre for European Policy Studies, Bruselas, febrero de 2015: http://www.ceps.eu/book/more-union-european-defence (última consulta 14/03/2015). 
de integración europea. La distinta percepción de las amenazas y cultura estratégica de los Estados miembros, las diferentes intenciones y preferencias, e incluso la falta de confianza mutua y solidaridad, son obstáculos que han bloqueado una integración mayor.

Aunque esas limitaciones han sido una constante desde el nacimiento de la Unión Europea (UE) en 1992, como se acreditará a lo largo de este documento, en la actualidad han ganado relevancia ante los cambios producidos en el entorno estratégico, ya que Europa se encuentra rodeada de un arco de inestabilidad que abarca desde el Este del continente con la crisis de Ucrania, hasta el Norte de África con el repunte del terrorismo de raíz islamista radical.

Sin ánimo de ser exhaustivos, sí que es preciso resumir los principales hitos que llevaron al nacimiento de la Política Europea de Seguridad y Defensa (PESD), como componente de la Política Exterior y de Seguridad Común (PESC), dado que el modo en que se desarrolló ese proceso está en la base de muchas de sus carencias actuales ${ }^{1}$.

Intentado superar el famoso aforismo según el cual Europa es un gigante económico, un enano político, y un gusano militar, las grandes expectativas creadas al finalizar la Guerra Fría se reflejan en la frase del ministro de exteriores luxemburgués, Jacques Poos, que en junio de 1991 afirmó que había llegado «la hora de Europa y no la de los americanos» para hacer frente al comienzo de las guerras en los Balcanes².

Sin embargo, la entonces Comunidad Europea carecía de capacidad para actuar ante una crisis de esa magnitud, de entrada por la manifiesta oposición de Estados Unidos al desarrollo de una defensa europea independiente de la OTAN (y por tanto fuera de su control). La presión de Washington sobre los aliados europeos fue constante en 1991 mientras se negociaba el Tratado de Maastricht, en particular para que la UE no integrase a la Unión Europea Occidental (UEO), alianza militar creada en 1948 que había permanecido a la sombra de la Alianza Atlántica durante décadas ${ }^{3}$.

${ }^{1}$ RUIZ, F., «El largo camino hacia un Ejército europeo», Documento de Análisis del IEEE, $\mathrm{n} .{ }^{\circ}$ 17/2010: $\mathrm{http}: / / \mathrm{www}$. ieee.es/Galerias/fichero/docs_analisis/2010/DIEEEA172010CaminoHaciaEjercitoEuropeo.pdf (última consulta 25/02/2015).

2 Declaraciones a 29 de junio de 1991, coincidiendo por tanto con la proclamación de la independencia de Croacia y Eslovenia, y en plena Guerra de los diez días en esta última república. «Conflict in Yugoslavia; Europeans send high-level team», The New York Times, 29 de junio de 1991: http://www.nytimes.com/1991/06/29/world/conflict-in-yugoslavia-europeanssend-high-level-team.html (última consulta 25/02/2015).

${ }^{3}$ El 4 diciembre de 1990 el subsecretario de estado de EEUU, Raymond Seitz, reiteró el interés de EEUU en el pilar europeo de seguridad y defensa que estaba surgiendo, pero advirtió en contra de cualquier estructura paralela que amenazase con «vaciar a la OTAN de su contenido y en consecuencia perder la justificación para una presencia estadounidense en Europa». BOZO, F., Mitterrand, the end of Cold War, and the German reunification, Berghahn Books, Nueva York, 2009, p. 337. 
En todo caso, los objetivos de EEUU se vieron favorecidos por la falta de coherencia de los entonces doce Estados miembros, ya que mientras el bloque europeísta (con Francia, Alemania y España) abogaba por la incorporación de la UEO como brazo armado de la Unión, el bloque atlantista (con Reino Unido, Países Bajos e Italia) prefería convertirla en el pilar europeo de la OTAN. La solución de compromiso fue el mantener a la UEO separada de la UE, aunque ésta podría recurrir a sus capacidades de considerarlo necesario ${ }^{4}$.

El hecho es que a pesar de que durante la guerra de Bosnia (1992-1995) fueron los europeos los que más contribuyeron al esfuerzo internacional por frenar el conflicto, lo hicieron en el marco de la ONU y de la OTAN, en lugar de en el de la UE. La percepción final fue que Europa era incapaz de resolver una crisis en su propio vecindario, y que sólo cuando EEUU decidió implicarse militarmente se puso fin a la guerra. El posterior Tratado de Ámsterdam de 1997 no tuvo mayor trascendencia en el ámbito de la PESC que la inclusión de las misiones Petersberg ${ }^{5}$, y la creación del Alto Representante para la PESC, cargo que recayó en el español Javier Solana.

La superación de ese impasse fue impulsada por el Reino Unido y Francia, que en su Cumbre bilateral de 1998 en Saint-Malo declararon que la Unión debía tener «la capacidad de actuar con autonomía, apoyada por una fuerza militar creíble, los recursos para usarla y la disposición de hacerlo, para responder a crisis internacionales» ${ }^{6}$. Esos postulados fueron asumidos por los restantes Estados miembros en el Consejo Europeo de Colonia en junio de $1999^{7}$.

4 «La Unión pide a la UEO, que forma parte integrante del desarrollo de la UE, que elabore y ponga en práctica las decisiones y acciones de la Unión que tengan repercusiones en el ámbito de la defensa». Tratado de la Unión Europea, Maastricht, p. 126: http://europa.eu/ eu-law/decision-making/treaties/pdf/treaty_on_european_union/treaty_on_european_union_ es.pdf (última consulta 25/02/2015).

${ }^{5}$ Las llamadas «misiones Petersberg» se habían definido en el marco de la UEO, e incluyen misiones humanitarias y de rescate, de mantenimiento de la paz y otras en las que intervengan fuerzas de combate para la gestión de crisis, incluidas las de restablecimiento de la paz, a desarrollar al amparo del capítulo VI de la Carta de la ONU. Por tanto, la Unión renunció a llevar a cabo misiones de imposición de la paz (peace enforcement) al amparo del capítulo VII de la Carta de la ONU. Declaración de Petersberg del Consejo de la UEO, Bonn, 19 de junio de 1992: http://www.weu.int/ (última consulta 25/02/2015).

${ }^{6}$ Declaración conjunta sobre la defensa europea, Saint-Malo, 4 de diciembre de 1998: http://www.atlanticcommunity.org/Saint-Malo\%20Declaration\%20Text.html (última consulta 25/02/2015).

7 Consejo Europeo de Colonia. Anexo III de las conclusiones de la presidencia. Informe de la presidencia sobre de la política europea común sobre seguridad y defensa, Colonia, 3-4 de junio de 1999: http://www.europarl.europa.eu/summits/kol2_es.htm\#bilIII (última consulta 25/02/2015). 
En este punto hay que mencionar de nuevo a EEUU, ya que su administración se opuso a estos avances, con la secretaria de estado Albright declarando que los europeos no debían duplicar capacidades ya existentes en la OTAN, ni discriminar a los aliados no pertenecientes a la UE (como Turquía), ni disgregar la seguridad europea de la norteamericana, posición bautizada como la «teoría de las $3 \mathrm{~d} »^{8}$.

\section{El «Objetivo Principal de Helsinki» y la relación con la OTAN}

Una vez tomada la decisión de crear la PESD en el Consejo Europeo de Colonia, la UE tuvo que hacer frente al hecho de que desde el fin de la Guerra Fría carecía de capacidades incluso para ejecutar acciones militares menores en el continente sin asistencia de EEUU, como consecuencia de los dividendos de la paz ${ }^{9}$. El intento de corregir esta situación comenzó en el Consejo Europeo de Helsinki, celebrado los días 10 y 11 de diciembre de 1999. El apartado II de las «Conclusiones de la Presidencia» se dedica a la PESD, y en él se reitera el propósito de la Unión de $^{10}$ :

Crear una capacidad de decisión autónoma y, en aquellas operaciones militares en las que no participe la OTAN en su conjunto, la capacidad de iniciar y llevar a cabo operaciones militares dirigidas por la UE en respuesta a crisis internacionales. Este procedimiento, que evitará duplicaciones innecesarias, no implica la creación de un ejército europeo.

En el anexo IV se especifican las capacidades militares con las que la UE tendría que dotarse para desarrollar las mencionadas misiones Petersberg: antes del año 2003, los Estados miembros debían estar en condiciones de desplegar un Cuerpo de Ejército (entre 50.000 y 60.000 efectivos), con las capacidades necesarias de mando, control, inteligencia, logística y otros

8 Albright aprovechó una rueda de prensa en el Cuartel General de la OTAN, pocas semanas después de la Cumbre franco-británica de Saint-Malo, para abordar esta y otras cuestiones clave para la seguridad europea. Ver Albright press conference at $N A T O H Q, 8$ de diciembre de 1998: http://www.fas.org/man/nato/news/1998/98120904_tlt.html (última consulta 25/02/2015).

${ }^{9}$ Denominación del proceso de los años 90 por el cuál parte de los presupuestos de defensa de Europa Occidental se reasignaron a gastos asociados al estado del bienestar, aprovechando la desaparición de la amenaza soviética. MINTZ, A. y HUANG, C., «Defense expenditures, economic growth, and the peace dividend», American Political Science Review, Vol. 84, n. ${ }^{\circ}$ 4, diciembre de 1990, pp. 1283-1293.

10 Conclusiones de la Presidencia, Consejo Europeo de Helsinki, 10 y 11 de diciembre de 1999: http://www.europarl.europa.eu/summits/hel1_es.htm\#b (última consulta 08/03/2015). 
servicios de apoyo a las operaciones de combate, además de contar con elementos aéreos y navales.

Esa Fuerza de Reacción Rápida se desplegaría en un plazo máximo de 60 días y se debía mantener en la zona al menos durante un año, para lo que precisaba una reserva adicional de unidades con menor grado de disponibilidad para sustituir a las fuerzas iniciales. Este nivel de ambición militar de la UE se denominó «Objetivo Principal de Helsinki» (Helsinki Headline Goal, $\mathrm{HHG})^{11}$.

Otro aspecto importante fue el desarrollo de las estructuras y capacidades civiles de gestión de crisis, ya que esto representa una diferencia básica entre la UE/PESD y la OTAN. En los Consejos Europeos de Santa María de Feira, en junio de $2000^{12}$, y Gotemburgo, en junio de $2001^{13}$, este objetivo se concretó en cuatro ámbitos prioritarios: policía, refuerzo del Estado de derecho, refuerzo de la administración, y protección civil ${ }^{14}$.

En lo referente al desarrollo de las capacidades militares para alcanzar lo establecido en el HHG, en otoño de 2000 el primer «Catálogo de Necesidades» identificó hasta 144 requerimientos concretos, agrupándolos en siete categorías: mando, control, comunicaciones e inteligencia; inteligencia, vigilancia, adquisición de objetivos y reconocimiento; capacidad de despliegue y movilidad; enfrentamiento efectivo; protección y supervivencia; sostenimiento y logística; y apoyo general.

Para cubrirlas, y como resultado de la «Conferencia de Compromisos de Capacidades» celebrada en Bruselas en noviembre de 2000, los quince

11 Ver The European Security and Defence Policy: from the Helsinki Headline Goal to the EU Battlegroups, Directorate-General for External Policies, 12 de septiembre de 2006: http://www.europarl.europa.eu/meetdocs/2009_2014/documents/sede/dv/sede030909noteesdp_/sede030909noteesdp_en.pdf (última consulta 08/03/2015).

${ }_{12}$ Conclusiones de la Presidencia, Consejo Europeo de Santa María da Feira, 19 y 20 de junio de 2000: http://www.europarl.europa.eu/summits/fei2_es.htm (última consulta 08/03/2015).

13 Conclusiones de la Presidencia, Consejo Europeo de Gotemburgo, 15 y 16 de junio de 2001: http://www.consilium.europa.eu/ueDocs/cms_Data/docs/pressData/es/ec/00200-r1.es1. pdf (última consulta 08/03/2015).

${ }^{14}$ Los Estados miembros debían ser capaces de desplegar hasta 5.000 agentes (1.000 de ellos en menos de un mes); hasta 200 jueces, fiscales y expertos en materias penitenciarias; un equipo de expertos para restablecer sistemas administrativos ineficaces; y en un plazo de entre tres y cinco horas, entre dos y tres equipos de evaluación con diez expertos en protección civil, y equipos de intervención compuestos por hasta 2.000 personas en el plazo de un mes. PÉREZ DE LAS HERAS, B. y CHURRUCA, C., Las capacidades civiles y militares de la UE: estado de la cuestión y propuestas de cara a la Presidencia Española 2010, Documento de Trabajo, n. ${ }^{\circ}$ 41, Fundación Alternativas, Madrid, 2009, p. 23: http://www.falternativas.org/opex/documentos/documentos-de-trabajo/las-capacidades-civiles-y-militaresde-la-ue-estado-de-la-cuestion-y-propuestas-de-cara-a-la-presidencia-espanola-2010 (última consulta 08/03/2015). 
Estados miembros procedieron a cuantificar sus contribuciones. Recogidas en el «Catálogo de Fuerzas», estas aportaciones sumaban más de 100.000 efectivos, 400 aviones y 100 buques, lo que en términos cuantitativos parecía cumplir el HHG. No obstante, se reconocía la necesidad de realizar mejoras cualitativas en las fuerzas ofrecidas, conforme a los requerimientos establecidos en el «Catálogo de Necesidades» ${ }^{15}$.

El siguiente eslabón, el llamado «Catálogo de Progreso de Helsinki», dejó establecidas en junio de 2001 las diferencias entre las contribuciones y las necesidades reales. Para solventar esas carencias, en el Consejo de Laeken de diciembre de 2001 se impulsó el denominado «Plan de Acción Europeo de Capacidades» (European Capabilities Action Plan, ECAP). A pesar de persistir las limitaciones, el Consejo de Asuntos Generales y Relaciones Exteriores declaró, en mayo de 2003, la plena capacidad operativa de la Fuerza de Reacción Rápida para toda la gama de misiones Petersberg.

Por otra parte, la falta de un reconocimiento mutuo y explícito de la contribución que correspondía hacer a la UE y a la OTAN a la seguridad euroatlántica se pretendió superar con la «Declaración Conjunta de la OTAN/UE sobre la PESD», aprobada en diciembre de 2002 bajo los principios de la búsqueda de una concertación eficaz, la igualdad entre organizaciones, la autonomía de decisión, el respeto a los principios de la Carta de la ONU, y el desarrollo coherente y transparente de las capacidades militares $^{16}$.

Para dar cumplimiento al último punto se constituyó en 2003 del «Grupo de Capacidades OTAN-UE», que coordina desde entonces las iniciativas relativas a sus capacidades militares, tomando como base la sinergia y la complementariedad, con objeto de reforzarse mutuamente. Esto representó un hito importante, ya que de otro modo los Estados que son miembros de ambas organizaciones debían cumplir, con sus limitados presupuestos de defensa, los diferentes requerimientos de una y otra.

La principal herramienta para la cooperación OTAN-UE son los acuerdos Berlín Plus ${ }^{17}$, en vigor desde el 17 de marzo de 2003. Estos

15 SOLANA, J., «Desafíos de la defensa europea», Política Exterior, n. ${ }^{0}$ 79, 2001, p. 73.

16 Remarks by Javier Solana, following the agreement on the establishment of EU-NATO permanent arrangements, Bruselas, 16 de diciembre de 2002: http://www.consilium.europa. eu/uedocs/cms_data/docs/pressdata/en/declarations/73803.pdf (última consulta 08/03/2015).

17 Sobre esta cuestión, ver EU-NATO: the Framework for permanent relations and Berlin Plus: http://www.consilium.europa.eu/uedocs/cmsUpload/03-11-11\%20Berlin\%20 Plus\%20press\%20note\%20BL.pdf; REICHARD, M., The EU-NATO relationship: A legal and political perspective, Ashgate Publishing, Burlington, 2006, pp. 273-310; STADELMALER, F., On the emergence of ESDP and EU-NATO cooperation, GRIN Verlag, 2006, pp. 57-71; y HAINE, J-Y., Berlin Plus, EUISS, París, 2007: http://www.peacepalacelibrary. nl/ebooks/files/03-jyhb\%252B.pdf (últimas consultas 08/03/2015). 
acuerdos sientan la base de la cooperación en el ámbito de la gestión de crisis, y permiten apoyar operaciones dirigidas por la UE en las que no participa la OTAN en su conjunto. Sus principios son: permitir el acceso de la UE a capacidades de planeamiento operativo de la OTAN; la disponibilidad de capacidades y medios comunes OTAN para su uso por la UE; la posibilidad de que el 2. ${ }^{\circ}$ SACEUR ${ }^{18}$ (un europeo) sea mando de una operación de la UE; el establecimiento de mecanismos de consulta; y la integración de las necesidades de la UE en el planeamiento de defensa de la OTAN.

En todo caso, es preciso destacar que el proceso de aprobación de Berlín Plus duró casi cuatro años, ya que aunque el punto 5 de la declaración del Consejo Europeo de Colonia de 1999 esbozaba las condiciones para la participación en las misiones de la UE de los Estados miembros no-aliados de la OTAN, las sucesivas propuestas al respecto no satisficieron los requerimientos de Turquía, que se opuso por tanto a que la UE pudiera acceder a las capacidades de la Alianza para la conducción de sus operaciones ${ }^{19}$.

El veto turco se superó en 2001, pero las concesiones realizadas por el llamado «Documento de Ankara» ${ }^{20}$ y el procedimiento ad-hoc de negociación empleado suscitaron el rechazo de Grecia, que amenazó con vetar los acuerdos en el ámbito de la UE. Ese bloqueo no se superó hasta la aprobación de la «Declaración del Consejo de 12 de diciembre de 2002 aneja a las conclusiones del Consejo Europeo de Copenhague» ${ }^{21}$. En ella, se establecía

18 SACEUR: Supreme Allied Commander in Europe.

19 «Turquía temía que una fuerza de la UE pudiese llegar a operar en sus áreas de interés, sin que ella tuviera el derecho de decidir o intervenir [...] Esos temores de exclusión la empujaron varias veces a vetar cualquier acuerdo que diese acceso a la UE a los medios militares y de planeamiento de la OTAN [...] Esto era una espada de doble filo, ya que aunque Turquía detuvo los planes de la UE para lanzar misiones militares antes de estar equipada para hacerlo autónomamente, también dio un incentivo a la UE para avanzar hacia una autonomía total con respecto a la OTAN». MEDINA-ABELLAN, M., Turkey, the European Security and Defence Policy, and accession negotiations, Working Paper, $\mathrm{n} .{ }^{\circ} 1$, SINAN, 24 de abril de 2009, pp. 2-3: http://sinan.ces.metu.edu.tr/dosya/miguelwp1.pdf (última consulta 08/03/2015).

20 «En la práctica, el Documento de Ankara suponía importantes concesiones desde el punto de vista de los europeos, obligando a la UE a un engorroso sistema de consultas que recordaba a la UEO y venía a añadirse a las consultas multilaterales con la OTAN, y utilizaba en algunos pasajes un lenguaje políticamente inapropiado en un Documento de la UE.» ACERETE, M., «La Alianza Atlántica y la Política Europea de Seguridad y Defensa», en VV.AA., El vínculo trasatlántico, Monografía del CESEDEN, n. ${ }^{\circ}$ 2, Madrid, julio de 2004, p. 91.

${ }^{21}$ Consejo Europeo de Copenhague. Anexo II de las Conclusiones de la Presidencia, Bruselas, 29 de enero de 2003: http://www.consilium.europa.eu/uedocs/cms_data/docs/ pressdata/es/ec/73843.pdf (última consulta 08/03/2015). 
que Berlín Plus sólo se aplicaría a los miembros de la UE que también fuesen aliados de la OTAN, o que al menos participaran en su «Asociación por la Paz», lo que automáticamente excluye a Chipre.

El 31 de marzo de 2003 la UE lanzó su primera operación militar, «EUFOR Concordia», para relevar a la operación «Allied Harmony» de la OTAN en la República de Macedonia. A esta operación, finalizada con éxito el 31 de diciembre de 2003, le siguió la trasferencia de la operación SFOR de la OTAN en Bosnia y Herzegovina a la operación «EUFOR Althea» de la UE, que comenzó el 2 de diciembre de 2004. Sin embargo, con esas dos operaciones finalizó la cooperación entre la OTAN y la UE utilizando el marco de Berlín Plus, inútil tras la entrada de Chipre en la Unión.

Aunque en ambos casos el balance fue positivo, se pueden matizar la eficacia real del modelo, ya que se produjo una transferencia directa de responsabilidad sobre la operación de la OTAN a la UE, por lo que quedó por demostrar que la puesta en marcha de una operación PESD con medios de la OTAN, allí donde la Alianza no estuviese previamente operando, se produjese con la misma facilidad ${ }^{22}$. Además, y aunque el planeamiento en el nivel militar-estratégico se realizó de un modo rápido y eficaz, en el nivel político-estratégico se vio dificultado por las continuas peticiones de información por parte de Turquía, a las que hubo de responder para evitar el veto de ese país, proceso que se prolongó seis meses.

Por otra parte, las relaciones entre EEUU y sus aliados europeos empeoraron notablemente durante la crisis que llevó a la invasión de Irak en marzo de 2003. Los miembros de la UE más opuestos a las políticas de Washington (Francia, Alemania, Bélgica, Luxemburgo) desafiaron en abril de 2003 el principio de la no-duplicación de capacidades, con la propuesta de creación de un Cuartel General (conocido por las siglas OHQ, Operational Headquarters) que proporcionase a la UE una capacidad de planeamiento y conducción de operaciones militares independiente de la OTAN.

Esa propuesta despertó, nuevamente, la firme oposición de EEUU. El 15 de octubre de 2003 su embajador ante la OTAN, Nicholas Burns, describió la política de la UE como «la amenaza más significativa al futuro de la OTAN». Esta reacción venía a reiterar la posición ambivalente de EEUU frente a la dimensión militar y estratégica de la UE, ya que una vez más se mostraba su apoyo al incremento de las capacidades militares de los europeos, pero estableciendo a la vez unas condiciones muy estrictas al alcance

22 SIMÓN, L., «Command and Control? Planning for EU military operations», EUISS Occasional Paper, n. ${ }^{\circ}$ 81, enero de 2010, pp. 30-31: http://www.iss .europa.eu/uploads/media/ Planning_for_EU_military_operations.pdf. (última consulta 08/03/2015). 
político de esos esfuerzos. Este paradójico trastorno bipolar estadounidense fue descrito por Nicole Gnessoto del siguiente modo ${ }^{23}$ :

Los americanos tienen dos visiones: o bien los europeos son considerados irrelevantes porque carecen de cualquier capacidad militar seria, o vistos como potencialmente peligrosos una vez que están políticamente organizados o son excesivamente ambiciosos. En un caso, son vistos como inútiles, una carga, o un condicionante; en el otro, como un competidor, una amenaza, o un traidor.

Aunque el Cuartel General de nivel estratégico-militar no llegó a crearse, ese mismo año 2003 se produjo un evento que supuso un notable hito en el desarrollo de la PESD, cuando el 30 de mayo el Consejo de Seguridad de Naciones Unidas aprobó la Resolución $1484^{24}$, que autorizaba el despliegue de una «Fuerza Multinacional Provisional de Emergencia» en la provincia congoleña de Bunia en apoyo de la Misión de la ONU en la República Democrática del Congo.

Francia, que desde el 15 de mayo negociaba con la ONU el asumir el liderazgo de esa fuerza, planteó convertirla en una operación militar de la UE, aprovechando la única alternativa a Berlín Plus: el uso de uno de los Cuarteles Generales que algunos Estados miembros habían puesto a disposición de la Unión ${ }^{25}$, en este caso el francés de Mont Valérien (París). El Consejo de la UE aprobó esa opción el 5 de junio, y el 12 de junio comenzó el despliegue de la «Operación Artemis».

La trascendencia de esta operación, que finalizó el 1 de septiembre de 2003, radica en que la decisión de la UE se tomó sin consultar con la OTAN, a pesar de que EEUU había interpretado en el proceso negociador de Berlín Plus que la Alianza Atlántica siempre podría adoptar la primera decisión, y que sólo si decidía no intervenir, la UE podría optar por hacerlo o no.

${ }^{23}$ GNESSOTO Nicole, «EU, US: visions of the world, visions of the other», en LINDSTROM, G. (ed.), Shift or Rift. Assessing US-EU relations after Iraq, EUISS, París, octubre de 2003,p. 33.

${ }^{24}$ Resolución 1484 del Consejo de Seguridad (2003), de 30 de mayo, sobre la situación relativa a la República Democrática del Congo: http://daccess-dds-ny.un.org/doc/UNDOC/ GEN/N03/377/71/PDF/N0337771.pdf?OpenElement (última consulta 08/03/2015).

25 Además de Francia con Mont Valérien, los Estados miembros que han ofrecido un OHQ a la UE son Alemania (Potsdam), Reino Unido (Northwood), Italia (Roma) y Grecia (Larissa). Se trata de una capacidad nacional que cuando se pone a disposición de la Unión se completa con personal militar de otros Estados miembros, y se desactiva al acabar la operación. Con ese sistema, se dificulta el planeamiento inicial, y se pierde al finalizar la experiencia operacional adquirida. 


\section{Avances de la PESD tras la aprobación de la Estrategia Europea de Seguridad}

El año 2003 concluyó con la aprobación en el Consejo Europeo de Bruselas, celebrado los días 12 y 13 de diciembre, de la Estrategia Europea de Seguridad (en adelante EES-2003), bajo el título de «Una Europa segura en un mundo mejor» ${ }^{26}$, conocida también como Documento Solana por el papel jugado por el Alto Representante en su elaboración, que vino a cubrir la falta de una estrategia conjunta que pudiese servir de marco de referencia para el desarrollo de la PESD ${ }^{27}$.

Con la EES-2003 como la guía estratégica de la que la UE había carecido durante los cinco años transcurridos desde Saint-Malo, la PESD inició en 2004 una etapa de gran desarrollo, en la que destacan el nacimiento de los «Grupos del Combate» (Battlegroups, BG) en febrero y la puesta en marcha de la Agencia Europea de Defensa (European Defence Agency, EDA) en julio.

En lo referido a los «Grupos de Combate» o agrupaciones tácticas ${ }^{28}$, cuando en el Consejo Europeo de Helsinki de 1999 se aprobó el HHG ya se hizo referencia a la necesidad de disponer de «elementos de respuesta rápida más pequeños, disponibles y desplegables con un alto grado de alistamiento» ${ }^{29}$. En la Cumbre franco-británica del 4 de febrero de 2003 se recuperó esa idea, ya que su declaración final destacaba la necesidad de que la UE dispusiera de «capacidades de planeamiento y de despliegue de fuer-

${ }^{26}$ Una Europa segura en un mundo mejor. Estrategia Europea de Seguridad, Bruselas, 12 de diciembre de 2003: http://www.consilium.europa.eu/uedocs/cmsUpload/031208ESSIIES.pdf. (última consulta 08/03/2015).

27 «El modo en que la PESD fue establecida creo un «desfase conceptual» que demandaba un trabajo posterior de estrategia, porque el énfasis inicial se puso mucho más en las capacidades y en los tipos genéricos de operaciones que en sus objetivos políticos y sus fundamentos». BAILES, A., «The European Security Strategy: An evolutionary history», SIPRI Policy Paper, n. ${ }^{\circ}$ 10, febrero de 2005, p. 5: http://books.sipri.org/files/PP/SIPRIPP10.pdf (última consulta 08/03/2015).

28 «Las agrupaciones tácticas son unidades de 1.500 efectivos. El procedimiento de la decisión y de planeamiento prevé que la UE sea capaz de iniciar una operación en los cinco días siguientes a la aprobación del Consejo, y que las fuerzas empiecen a llevar a cabo su misión en un plazo máximo de diez días, pudiendo sostenerse en la zona entre 30 y 120 días». Desde enero de 2007 la UE mantiene al menos dos BG por semestre en alerta, pero hasta la fecha ninguno ha sido desplegado. Ver LINDSTROM, G., «Enter the EU Battlegroups», Chaillot Paper, n. ${ }^{\circ}$ 97, EUISS, febrero de 2007: http://www.iss.europa.eu/uploads/media/cp097.pdf (última consulta 08/03/2015).

${ }^{29}$ Conclusiones de la Presidencia. Consejo Europeo de Helsinki, Anexo IV «Informes de la Presidencia al Consejo Europeo de Helsinki sobre «El refuerzo de la Política Europea Común de Seguridad y Defensa» y sobre «La gestión no militar de crisis de la Unión Europea»: http://www .europarl.europa.eu/summits/hel2_es.htm\#IV (última consulta 08/03/2015). 
zas en un corto periodo de tiempo, incluyendo un despliegue inicial de fuerzas terrestres, aéreas y navales entre 5 y 10 días» ${ }^{30}$.

La rapidez del despliegue de la operación «Artemis» en junio de 2003 reafirmó lo necesario del concepto, que fue respaldado por Alemania en febrero de $2004^{31}$ y oficialmente aprobado en el Consejo Europeo de ministros de exteriores y defensa de los días 17 y 18 de mayo del mismo año. En el Consejo Europeo de Bruselas de los días 17 y 18 de junio de 2004 se decidió integrar los BG en el nuevo «Headline Goal 2010», objetivo de fuerza de la UE que sustituyó al HHG-200332.

En lo referido a la Agencia Europea de Defensa, esta fue creada por la Acción Común 2004/551/PESC del Consejo Europeo ${ }^{33}$. Bajo la autoridad del Consejo y abierta a la participación de todos los Estados miembros, la EDA tiene por objetivo el impulso de las capacidades de defensa en el ámbito de la gestión de crisis, el fomento y la intensificación de la cooperación europea en materia de armamento, el fortalecimiento de la «Base Tecnológica e Industrial de la Defensa» europea y la creación de un mercado europeo competitivo ${ }^{34}$.

${ }^{30}$ Franco-British summit. Declaration on strengthening European cooperation in security and defence, Le Touquet, 4 de febrero de 2003: http://pure.au.dk/portal/files/7371/bilag nummer_2.doc (última consulta 08/03/2015).

31 «The battlegroups concept - UK/France/Germany food for thought paper», Chaillot Paper, Vol. V, n. ${ }^{\circ}$ 75, EUISS, Paris, 1 de febrero de 2005, p. 10: http://www.iss.europa.eu/ uploads/media/cp075e.pdf (última consulta 08/03/2015).

32 «Frente al planteamiento más cuantitativo del Headline Goal de Helsinki, el nuevo Objetivo introduce un enfoque más cualitativo con respecto a la organización de las fuerzas, incidiendo así en la necesidad de una mayor planificación militar y en un mejor uso de los recursos compartidos y puestos en común». PÉREZ DE LAS HERAS, B. y CHURRUCA, C., op. cit., nota 15 , p. 16.

Sobre el nuevo objetivo de fuerzas y los Grupos de Combate, ver también LINDLEYFRENCH, J., Headline Goal 2010 and the concept of the European Union Battlegroups: An assesment of the build-up of a European Defence capability, Cicero Foundation, París, diciembre de 2005: http://www.cicerofoundation.org/pdf/lecture_lindleyfrench_dec05.pdf (última consulta 08/03/2015).

33 «Acción Común 2004/551/PESC del Consejo de 12 de julio de 2004 relativa a la creación de la Agencia Europea de Defensa», Diario Oficial de la Unión Europea, n. ${ }^{\circ}$ L 245, 17 de julio de 2004: http://eur-lex.europa.eu/LexUriServ/site/es/oj/2004/1_245/ 1_24520040717es00170028.pdf. (última consulta 08/03/2015).

34 Sobre la EDA, ver VV.AA., »La Agencia Europea de Defensa: pasado, presente y futuro», Monografía del CESEDEN, n. ${ }^{\circ}$ 107, enero de 2009: http://www.defensa.gob.es/ ceseden/Galerias/destacados/publicaciones/monografias/ficheros/107_LA_AGENCIA_ EUROPEA_DE_DEFENSA_PASADO_PRESENTE_Y_FUTURO.pdf. (última consulta 08/03/2015). 
La EDA publicó en octubre de 2006 la llamada «Visión a Largo Plazo» (Long Term Vision, LTV) ${ }^{35}$, informe elaborado para servir de guía a los encargados del planeamiento de la defensa, de modo que en un horizonte de 20 años se puedan cubrir los requerimientos de la PESD. En base a la LTV, en julio de 2008 los 26 Estados miembros de la Agencia (todos los de la UE excepto Dinamarca, voluntariamente excluida) aprobaron el «Plan de Desarrollo de Capacidades» (Capabilities Development Plan, CDP $)^{36}$, que define las necesidades y prioridades militares futuras de la PESD. El CDP contempla 24 áreas de actuación, y absorbió el ECAP de 2001.

La opción de utilizar como OHQ uno de los que los Estados miembros ofrecen a la UE, puesta en práctica por primera vez en 2003 con Artemis, se volvería a emplear en una segunda operación PESD en la República Democrática del Congo, en la que también se pusieron de manifiesto los inconvenientes del esta alternativa, ya que cuando en diciembre de 2005 el Departamento de Operaciones de Mantenimiento de la Paz de la ONU pidió por escrito el lanzamiento de una operación de apoyo a MONUC en el proceso electoral previsto para el verano de 2006, la UE se topó con serias dificultades a pesar del amplio margen de tiempo para llevar a cabo el planeamiento ${ }^{37}$.

Lo que ocurrió fue que en ese caso Francia, que nuevamente aportaba la mayor parte de las tropas, consideró que el OHQ debía ser ofrecido por otro Estado. En aquel momento el Reino Unido estaba implicado en Irak y Afganistán, e Italia afrontaba un periodo electoral, por lo que todas las miradas se volvieron hacia Alemania y su Cuartel de Potsdam. Las reticencias alemanas se pusieron de manifiesto al negarse a llevar a cabo el planeamiento hasta que Potsdam fuese oficialmente designado y las naciones asegurasen la asignación de fuerzas, lo que produjo un impasse de un mes en el proceso, y la UE no habría estado en condiciones de lanzar EUFOR RDC si no se hubiese producido finalmente un retraso en la fecha de las elecciones ${ }^{38}$.

Como consecuencia de esos problemas, la UE inició el llamado Proceso de Wiesbaden, que finalizó a mediados de 2007 con la decisión de reorga-

${ }^{35}$ An initial Long-Term Vision for European Defence capability and capacity needs, Agencia Europea de Defensa, 3 de octubre de 2006: http://www.eda.europa.eu/docs/documents/Long-Term_Vision_Report (última consulta 08/03/2015).

${ }^{36}$ Sobre el CDP, ver Agencia Europea de Defensa, http://www.eda.europa.eu/docs/documents/brochure_cdp.pdf (última consulta 08/03/2015).

37 SIMÓN, L., «Command and Control? Planning for EU military operations», EUISS Occasional Paper, n..$^{\circ}$ 81, París, enero de 2010, pp. 31-34: http://www.iss.europa.eu/uploads/ media/Planning_for_EU_military_operations.pdf (última consulta 08/03/2015).

38 Sobre EUFOR RDC, ver MATTELAER, A., «EUFOR RDC and the development of ESDP», en Towards Peace and Security in Central Africa. International Intervention in Africa. NATO and ESDP, Studia Diplomatica, Vol. 60, n. . 3, 2007, pp. 73-90. 
nizar el Estado Mayor de la UE para incluir en él una división dedicada al planeamiento, la MAP (Military Assesment on Planning). El Reino Unido prefirió no oponerse a la iniciativa al estar aislado en esta ocasión en el Consejo. A pesar de la limitada entidad cuantitativa de la iniciativa (la MAP sólo cuenta con diez oficiales en permanencia), cualitativamente supuso un paso adelante muy importante, al servir para evitar los tiempos muertos y facilitar la transición del planeamiento una vez se designa el $\mathrm{OHQ}^{39}$.

Este fue el modelo seguido en 2007 para EUFOR Chad-RCA ${ }^{40}$, ambiciosa operación militar con el despliegue de 3.700 efectivos en una zona de muy complejo apoyo logístico, usando una vez más el OHQ francés de Mont-Valérien. El respaldo legal fue la Resolución 1778 del Consejo de Seguridad $^{41}$, y se concibió como una operación complementaria a la Misión de las Naciones Unidas en la República Centroafricana y el Chad (MINURCAT).

\section{Las lecciones aprendidas y el fallido proyecto constitucional}

Toda esa sucesión de eventos relacionados con el desarrollo de capacidades y el planeamiento y conducción de operaciones ilustran las limitaciones prácticas de la PESD, y permiten llegar a la conclusión de que a pesar de que en este periodo la UE actuó cada vez con una mayor independencia estratégica con respecto a la OTAN, no se produjo un pleno desarrollo de las estructuras de gestión de crisis necesarias, debido a las diferencias internas entre los Estados miembros más europeístas (como Francia), los más atlantistas (como el Reino Unido) y los partidarios de un papel militar limitado para la UE (como Alemania).

Pero esa no fue la única lección aprendida en ese periodo. Otro de los problemas era que las estructuras de gestión de crisis y las capacidades militares y civiles se habían desarrollado en paralelo y con una limitada interacción, cuando precisamente la característica distintiva de la UE con res-

39 Ver NESTIER, C., «Post Wiesbaden: Improving Strategic Planning», en New Missions, new challenges, Impetus, n. $^{\circ}$ 6, primavera/verano de 2008, pp. 8-10: http://www.consilium.europa.eu/uedocs/cmsUpload/IMPETUS\%205.pdf (última consulta 08/03/2015).

40 Sobre EUFOR TChad-RCA, ver MATTELAER, A., «The Strategic Planning of EU Military. Operations-The Case of EUFOR TCHAD/RCA», IES Working Paper, n. ${ }^{\circ}$ 5, 2008: http://www.ies.be/files/IES\%20working\%20paper\%205_Alexander\%20Mattelaer.pdf (última consulta 08/03/2015).

${ }^{41}$ Resolución 1778 del Consejo de Seguridad de Naciones Unidas (2007), de 25 de septiembre, sobre la situación en el Chad, la República Centroafricana y la subregión: http:// daccess-dds-ny.un.org/doc/UNDOC/GEN/N07/516/18/PDF/N0751618.pdf?OpenElement (última consulta 08/03/2015). 
pecto a otras organizaciones, como la OTAN, era su capacidad de disponer de un completo catálogo de herramientas.

Así por ejemplo, existían dos Comités independientes para asesorar al Comité Político y de Seguridad (COPS), el Comité Militar y el Comité para los Aspectos Civiles de Gestión de Crisis (CIVCOM), y lo mismo se reproducía en los órganos de trabajo de la Secretaría General del Consejo, donde dentro de la Dirección General de Asuntos Exteriores (DG E) existían por separado una Dirección de Defensa (DGE VIII) y una Dirección para Gestión Civil de Crisis (DGE IX), apoyadas respectivamente por el Estado Mayor y por la Capacidad de Planeamiento y Conducción Civil (CPCC $)^{42}$.

La solución a todas estas cuestiones se confió al proyecto de elaboración de una Constitución para la $\mathrm{UE}^{43}$, que fue aprobada por el Consejo Europeo el 18 de junio de 2004. En el ámbito de la PESD, el proyecto constitucional incluía innovaciones como: el nombramiento de un Ministro de Asuntos Exteriores que dirigiese todos organismos de la Comisión y del Consejo con competencias en la acción exterior, unificados en un ente único; un nuevo compromiso de solidaridad por el que los Estados miembros se prestarían asistencia en caso de un ataque terrorista o de una catástrofe natural; y una cláusula de defensa colectiva que pudiese reemplazar definitivamente al art. 5 del Tratado de Bruselas modificado de la UEO ${ }^{44}$.

Además, la Constitución contenía también una cuestión clave para el desarrollo de las capacidades de gestión de crisis que la UE precisaba, las llamadas «Cooperaciones Estructuradas Permanentes» (CEP). Con esa herramienta, un grupo de Estados miembros que dispusieran de un nivel adecuado de gasto de defensa, tomasen pasos concretos para reforzar la disponibilidad, interoperabilidad, flexibilidad y capacidad de despliegue de sus fuerzas, y acometiesen proyectos para cubrir las carencias identificadas en el ECAP, podrían cooperar en grupos de pioneros, ayudando a los Estados

42 GREVI, G., «ESDP Institutions», en GREVI, G., HELLY, D. y KEOHANE, D. (eds.), European Security and Defence Policy. The first 10 years (1999-2009), EUISS, París, octubre de 2009, pp. 19-68: http://www.iss.europa.eu/uploads/media/ESDP_10-web.pdf (última consulta 08/03/2015).

${ }^{43}$ Sobre esta cuestión, ver CHORNET, R. (coord.), La Política de Seguridad y Defensa en el Tratado Constitucional, Tirant lo Blanc, Valencia, 2005; y SERRA, F., «La Constitución Europea y la PESC», Análisis del Real Instituto Elcano, n. ${ }^{\circ}$ 131, julio de 2004: http:// www.realinstitutoelcano.org/wps/portal/rielcano/contenido?WCM_GLOBAL_CONTEXT=/ elcano/elcano_es/zonas_es/europa/ari+131-2004 (última consulta 08/03/2015).

${ }^{44}$ BRADY, H. y BARYSCH, K., «The CER's Guide to the EU's Constitutional Treaty», CER Policy Brief, Londres, julio de 2004: http://www.proyectos.cchs.csic.es/euroconstitution/Treaties/Library\%20(Since\%20June\%202007/Brady\%202007b.pdf (última consulta 08/03/2015). Sobre la cláusula de defensa mutua, ver BERMEJO, R., «La cláusula de defensa mutua: ¿Un paso adelante hacia una auténtica Política de Seguridad y Defensa?», en CHORNET Ramón (coord.), op. cit., nota 44, pp. 71-94. 
con menores capacidades a especializarse y a poner en común sus recursos nacionales ${ }^{45}$.

En todo caso, el fracaso en 2005 del proceso de ratificación del Tratado Constitucional, tras el no en referéndum de Francia (29 de mayo) y los Países Bajos (1 de junio), dejó en suspenso sus innovaciones hasta la entrada en vigor del Tratado de Lisboa el 1 de diciembre de 2009. Sin embargo, cabe destacar que la PESD continuó su desarrollo a pesar de la falta de una referencia de nivel superior: además de las cinco operaciones militares ya mencionadas, hasta el 1 de enero de 2008 la UE inició un total de trece misiones civiles.

\section{El desarrollo y optimización de las capacidades militares}

En la EES-2003 se afirma que «para que nuestros ejércitos se conviertan en fuerzas móviles más flexibles, capaces de hacer frente a las nuevas amenazas, serán necesarios mayores recursos para la defensa y un uso más eficaz de dichos recursos» ${ }^{46}$. Ese uso eficaz, según la propia Estrategia, precisa de la eliminación de duplicaciones innecesarias por medio del recurso sistemático a capacidades compartidas (Sharing) y puestas en común (Pooling), conceptos que se definen del siguiente modo ${ }^{47}$ :

- Sharing: uno o más países proporcionan a sus socios una capacidad o llevan a cabo una misión en beneficio de otros Estados. Si esto se realiza de un modo sistemático, algunos países pueden prescindir de esa capacidad y ahorrar costes. Por ejemplo, Alemania lleva a cabo la vigilancia marítima del Mar del Norte, eximiendo a Países Bajos de esa responsabilidad.

- Pooling: en este caso, las capacidades nacionales también se proporcionan a otros países, pero en el marco de una estructura multinacional que agrupa las diversas contribuciones y coordina su empleo. Esto se puede aplicar al desarrollo, la obtención y la operación de nuevas capacidades, lo que permite a un país o bien obtener un mayor número de unidades, o acceder a una capacidad avanzada que no podría obtener por su cuenta por su excesivo coste.

45 HAINE, J-Y., «ESDP transformed?», en Examining NATO's transformation, NATO Review, n. ${ }^{\circ}$ especial, primavera de 2005, p. 28: http://www.nato.int/docu/review/2005/issue2/ english/military.html (última consulta 08/03/2015).

46 Una Europa segura..., op. cit., nota 27, p. 12.

47 MÖLlING, C., «Pooling and Sharing in the EU and NATO», SWP Comments, n. ${ }^{\circ} 18$, junio de 2012, pp. 1-2: http://www.swp-berlin.org/fileadmin/contents/products/ comments/2012C18_mlg.pdf (última consulta 13/03/2015). 
Es evidente que este concepto del Pooling \& Sharing (en adelante $\mathrm{P} \& \mathrm{~S}$ ) es un modo de ahorrar dinero, en una etapa de recortes presupuestarios en Europa, y de potenciar el uso eficaz de los limitados recursos disponibles. Pero no se trata de una opción exenta de inconvenientes y riesgos, siendo el más evidente que una excesiva especialización de funciones supone abandonar el control nacional sobre determinados nichos de capacidades, para cuyo uso se pasa a depender de otros países. Ese difícil balance entre la eficiencia y la soberanía nacional dista mucho de haber sido resuelto, al igual que persisten las reticencias nacionales a levantar las barreras proteccionistas del mercado de la defensa de cada Estado miembro.

Por otra parte, mientras se producían todos esos avances de la PESD la Unión no había fijado de un modo explícito su nivel de ambición en el ámbito militar, factor básico y clave para cualquier planeamiento de la defensa. Esa carencia se intentó cubrir con la Declaración del Consejo Europeo de diciembre de $2008^{48}$, en la que se decía que además del objetivo inicial de desplegar la fuerza de 60.000 efectivos en 60 días para una gran operación, la UE debería ser capaz de llevar a cabo simultáneamente:

- Dos operaciones principales de estabilización y reconstrucción, de hasta 10.000 efectivos, con un componente civil (hasta 2 años).

- Dos operaciones de respuesta rápida con los BG de duración limitada.

- Una operación de evacuación de no-combatientes (menos de 10 días).

- Una misión de vigilancia/interdicción aérea o marítima.

- Una operación cívico/militar de asistencia (hasta 90 días).

- Hasta doce misiones menores civiles, más una misión con 3.000 expertos.

Para cumplir con esos requisitos operativos, la EDA lanzó diversos programas en cuatro áreas concretas: la proyección de fuerzas, incluyendo la modernización de helicópteros y la flota europea de transporte aéreo; la inteligencia basada en el espacio, con el programa Helios II de observación; la protección de las fuerzas desplegadas, con vehículos aéreos no tripulados; y la interoperabilidad, que incluyó un intercambio de alumnos de las academias militares de los Estados miembros.

48 Consejo Europeo de Bruselas 11 y 12 de diciembre de 2008. Conclusiones de la Presidencia: http://eurored.ccoo.es/comunes/recursos/99999/doc12627_Consejo_Europeo_de_ Bruselas_11_y_12_de_diciembre_2008._Conclusiones_de_la_Presidencia.pdf (última consulta $08 / 03 / 2015)$. 


\section{La entrada en vigor del Tratado de Lisboa: expectativas defraudadas}

El 1 de diciembre de 2009 entró en vigor el Tratado de Lisboa, que en el ámbito de la PESC recogía todas las herramientas incluidas en la fallida Constitución. Con ellas, la UE se encontraba en condiciones de asumir el papel como actor global que corresponde a su poder económico, político, e incluso militar, ya que sus entonces 27 miembros disponían de casi dos millones de soldados, e invertían en defensa una cantidad próxima a los 200.000 millones de euros. Sin embargo, desde entonces la evolución de la nueva PCSD ha sido en general decepcionante ${ }^{49}$.

En lo relativo a la Cooperación Estructurada Permanente, cinco años más tarde no se ha puesto en marcha ninguna. Sin embargo, Francia y el Reino Unido suscribieron en 2010 un amplio acuerdo bilateral de colaboración, ejemplo de las iniciativas que se podrían adoptar con las nuevas herramientas de Lisboa, pero que se ha llevado a cabo fuera del marco de la Unión, ignorando a los restantes países.

Cuando en 2011 surgió la crisis de Libia, la UE adoptó un perfil bajo y el papel de Ashton fue muy cuestionado. En todo caso, difícilmente puede la Alta Representante hablar con una sola voz, cuando los principales miembros de la UE adoptan posiciones opuestas, con Francia y el Reino Unido votando a favor de la intervención militar en el Consejo de Seguridad, y Alemania negándose a participar en las operaciones.

Además, desde 2008 la grave crisis financiera ha focalizado casi por completo la atención, dejando poco margen a cuestiones como la PCSD. La necesidad de reducir el déficit ha tenido como primera víctima a los presupuestos de defensa, con el agravante de que el nivel de partida ya era muy bajo, y de que esos recortes se han realizado con una óptica nacional, sin coordinación entre Estados miembros. Esa situación de estancamiento se prolongó, a grandes rasgos, hasta el año 2012.

\section{El Consejo Europeo de diciembre de 2012}

En el Consejo Europeo de diciembre de 2012 en Bruselas, los jefes de Estado y de gobierno acordaron «iniciar sus labores relativas al mayor desarrollo de la PCSD de la Unión Europea», de modo que en el Consejo de

49 RUIZ, F., «El papel como actor global de la Unión Europea desde la entrada en vigor del Tratado de Lisboa», Documento FUNCIVA, n. ${ }^{\circ}$ 98, abril de 2011: http://www funciva.org/ uploads/ficheros_documentos/1305731492_el_papel_como_actor_global_de_la_ue.pdf (última consulta 13/03/2015). 
diciembre de 2013 se pudiesen examinar los avances realizados, evaluar la situación y dar orientaciones, en particular fijando prioridades y plazos, para garantizar la eficacia de los esfuerzos realizados para que Europa esté a la altura de sus responsabilidades en materia de Seguridad ${ }^{50}$. Para todo ello, se fijaron tres líneas principales de acción:

- El aumentar la eficacia, visibilidad e impacto de la PCSD: con los mecanismos de prevención de conflictos, gestión de crisis y estabilización, y de respuesta a los retos emergentes; y con el refuerzo de la capacidad para el despliegue rápido y eficaz de las capacidades civiles y militares de gestión de crisis.

- La mejora de las capacidades de defensa: con la identificación de las actuales duplicaciones y carencias, y el establecimiento de prioridades de obtención; la cooperación sistemática y a largo plazo en materia de defensa, poniendo en común y compartiendo capacidades (una vez más, $\mathrm{P} \& \mathrm{~S}$ ); y propiciando las sinergias entre todas las iniciativas en marcha, tanto internas de la UE como con la «Defensa Inteligente» de la OTAN.

- El fortalecimiento del sector de la industria de defensa europea: con el desarrollo de una base tecnológica e industrial de la defensa más integrada, sostenible, innovadora y competitiva; y el establecimiento de mayores sinergias entre el I+D de los ámbitos civil y militar, la aplicación efectiva de las directivas relativas a la contratación pública y a las trasferencias en el interior de la UE.

Además, se invitaba a la Alta Representante para la PESC y a la Comisión Europea a que, en el ámbito de sus competencias, elaborasen propuestas y medidas adicionales para reforzar la PCSD y mejorar la disponibilidad de las capacidades civiles y militares necesarias, informando al respecto antes de finales de septiembre de 2013.

\section{El informe de la Comisión Europea sobre la PCSD}

El informe de la Comisión se aprobó el 24 de julio de 2013, bajo el título de «Hacia un sector de la defensa y la seguridad más competitivo y eficaz» ${ }^{51}$, con un fuerte foco en la tercera línea de acción fijada por el Con-

50 Conclusiones del Consejo Europeo (EUCO 205/12), Bruselas, diciembre de 2012: http://www.consilium.europa.eu/uedocs/cms_data/docs/pressdata/es/ec/134371.pdf (última consulta 13/03/2015).

51 Towards a more competitive and efficient defence and security sector, Bruselas, 24 de julio de 2013: http://europa.eu/rapid/press-release_MEMO-13-722_en.htm (última consulta 13/03/2015). 
sejo Europeo, la del fortalecimiento de la industria europea de defensa (que no es parte de nuestro objeto de estudio). En relación a las otras dos líneas de acción destacan las siguientes ideas-fuerza:

- Las capacidades militares son tecnológicamente complejas y caras, lo que en un escenario de restricciones presupuestarias dificulta a los Estados miembros el dotar adecuadamente a sus Fuerzas Armadas.

- De 2001 a 2010 el gasto de defensa de la UE bajó de 251.000 millones de $€$ a 194.000 millones; de 2005 a 2010 los presupuestos de I+D bajaron un $14 \%$ hasta los 9.000 millones de $€$. Estados Unidos invierte cada año en I+D militar siete veces más que los 28 miembros de la UE juntos.

- La baja inversión se agrava por la fragmentación del mercado europeo, que conduce a duplicaciones innecesarias. Un 75\% del gasto se ejecuta en clave nacional, sin cooperar con otros Estados miembros. Por ejemplo, en Europa existen 16 clases de fragatas, frente a una sola en EEUU.

Además, la Comisión realiza en la introducción una reflexión sobre el papel de la UE, ya que aunque la defensa está en el corazón de la soberanía nacional y las decisiones sobre las capacidades militares corresponden a los estados, éstos se han comprometido a fortalecer la acción exterior de la Unión mediante el desarrollo de capacidades civiles y militares para la prevención de conflictos y gestión de crisis.

\section{El informe de la Alta Representante PESC sobre la PCSD}

Por su parte, la Alta Representante PESC Catherine Ashton hizo público su informe el 15 de octubre de $2013^{52}$. Además de valorar el actual contexto estratégico y evaluar la situación de la PCSD, se detallan las propuestas y acciones para fortalecerla, agrupadas en las líneas establecidas por el Consejo ${ }^{53}$ :

- Incrementar la efectividad, visibilidad e impacto de la PCSD: desarrollar el enfoque integral para la prevención de conflictos, ges-

52 Final Report by the High Representative/Head of the EDA on the Common Security and Defence Policy, Bruselas, octubre 2013: http://eeas.europa.eu/statements/ docs/2013/131015_02_en.pdf (última consulta 13/03/2015).

53 Ver LABORIE, M., «Preparando el Consejo Europeo de diciembre de 2013: Informe sobre la PCSD», Documento Informativo del IEEE, n. ${ }^{\circ 27,} 28$ de octubre de 2013: http://www. ieee.es/Galerias/fichero/docs_informativos/2013/DIEEEI27-2013_Informe_PCSD_MLI.pdf (última consulta 13/03/2015). 
tión de crisis y estabilización; trabajar con los socios (OTAN, ONU, Unión Africana, etc.); establecer una seguridad en red; incrementar la capacidad de gestionar los retos marítimos y fronterizos; permitir el despliegue de los medios requeridos en el amplio espectro de operaciones de gestión de crisis; e incrementar la prevención de conflictos y la gestión del postconflicto.

- Potenciar el desarrollo de capacidades: permitir una cooperación europea en defensa más sistemática y a largo plazo (mediante el P\&S); focalizarse en el suministro de capacidades clave; facilitar las sinergias entre las iniciativas bilaterales, subregionales, europeas y multilaterales (en el marco de la EDA); y desarrollar las capacidades civiles de gestión de crisis.

En resumen, el informe considera que «la paz y la seguridad de Europa ha sido siempre un prerrequisito para su bienestar económico; ahora necesitamos evitar que las dificultades económicas de Europa afecten a su capacidad de afrontar los desafíos para la seguridad y la defensa» ${ }^{54}$.

\section{El Consejo Europeo de diciembre de 2013}

Con esos dos informes como base para el debate se celebró el Consejo Europeo los días 19 y 20 de diciembre de 2013, supuestamente monográfico sobre la PCSD aunque al final la omnipresente crisis económica se coló en la agenda. De hecho, de los 50 puntos de las Conclusiones, 23 están dedicados a la economía. Regresando al esquema de las tres líneas principales de acción fijadas en el Consejo de diciembre de 2012, en esta ocasión se citan logros y se establecen objetivos concretos.

Así por ejemplo, en lo relativo a la efectividad, visibilidad e impacto de la PCSD, se menciona que la UE despliega más de 7.000 efectivos en 12 misiones y cuatro operaciones; tiene una capacidad única de combinar todo tipo de herramientas (diplomacia, seguridad y defensa, finanzas, comercio, desarrollo y justicia); va a estudiar la mejora de la financiación de las misiones; y va a aprobar a lo largo de 2014 una Política Marco de Ciberdefensa y una Estrategia de Seguridad Marítima.

En lo relativo al desarrollo de capacidades, la cooperación multinacional es la única vía para mantener las capacidades clave, remediar las carencias, y evitar redundancias, de modo que los estados hagan un uso eficiente de los recursos y se asegure la interoperabilidad con otros actores clave como la OTAN. En concreto, se citan programas de obtención como el de

${ }^{54}$ Final Report by..., op. cit., nota 53, p. 4. 
sistemas aéreos no-tripulados, la capacidad de reabastecimiento en vuelo, o las comunicaciones por satélite.

El Consejo también aboga por incrementar la trasparencia y el intercambio de información sobre el planeamiento nacional de defensa de cada Estado miembro, y por reproducir el modelo de cooperación del Mando Europeo de Transporte Aéreo. Asimismo, se saluda el progreso alcanzado en la cooperación siguiendo el código de conducta de la EDA sobre el P\&S, y se destaca la necesidad de implementar plenamente el «Plan de Desarrollo de Capacidades Civiles» de gestión de crisis.

Todo este proceso ha provocado un nuevo debate sobre el papel de la UE en la Seguridad mundial. Se podría decir que la realidad de los hechos desborda el modelo establecido en el Consejo de diciembre de 2013, basado en dar pequeños pasos en el desarrollo de la PCSD siguiendo el viejo aforismo de para ir deprisa, ve solo, pero para llegar lejos, ve acompañado.

Resulta evidente que el entorno no respeta ese deseo de la UE de llegar lejos y unidos pero a paso lento, por lo que siempre parece ir a remolque de las circunstancias. Aunque esa actitud reactiva era la prevista incluso por los padres fundadores de la integración europea ${ }^{55}$, en la actualidad consideramos prioritario adoptar un enfoque top-down para dar un auténtico impulso a la PCSD.

\section{V. Últimas reflexiones y perspectivas de futuro}

La EDA recopila anualmente los datos sobre gasto en defensa proporcionados por los respectivos Ministerios ${ }^{56}$. En lo relativo a las inversiones, en 2007 la Agencia fijó cuatro umbrales de referencia (de aplicación voluntaria):

- Que un 20\% del total del gasto de defensa se dedique a adquisiciones.

- Que un 35\% de las adquisiciones se realicen por cooperación entre estados.

- Que un $2 \%$ del total del gasto de defensa se dedique a I+D.

- Que el 20\% del I+D se realice por cooperación entre Estados.

Los datos correspondientes a 2012 muestran que desde 2006 el porcentaje del gasto correspondiente a personal ha caído del $55 \%$ al $50,5 \%$, lo que

55 Como el francés Jean Monnet, que afirmaba que «Europa será impulsada por las crisis, y será la suma de las soluciones adoptadas para esas crisis». More Union in..., op. cit., nota 1, p. 1.

56 Defence Data 2012, EDA, 2013: http://www.eda.europa.eu/docs/default-source/edapublications/defence-data-booklet-2012-web (última consulta 13/03/2015). 
se ha conseguido sobre todo por la fuerte reducción (25\%) del número de efectivos militares, de 1.940 .000 en 2006 a 1.453 .000 en 2012.

El gasto de operación y mantenimiento ha subido del $21,6 \%$ al 23,7\%, y el de adquisiciones ha pasado del $19,6 \%$ al $20,4 \%$. Así se cumple la recomendación general de dedicar al menos el $20 \%$ a adquisiciones. Por su parte, el I+D ha sufrido una acusada caída (de 9.790 millones de $€$ en 2006 a 4.810 millones en 2012), aunque sigue representando un $2,5 \%$ del total.

Sin embargo, las inversiones se realizan cada vez más en clave nacional. Si en 2006 el 22,9\% de la inversión fue en cooperación interestatal, en 2012 ese porcentaje había bajado al 17,6\%, por debajo del mínimo de referencia. En el caso del I+D la situación es mucho peor, ya que se ha pasado de un $14,5 \%$ en 2006 a tan solo un 10,7\% en 2012.

En lo referente al número de tropas desplegadas en operaciones en el exterior, ha bajado en términos absolutos de los 83.300 de 2006 a los 49.500 de 2012, y en términos relativos del $4,3 \%$ al 3,4\%, muy lejos del objetivo de referencia del $10 \%$. Por todo lo expuesto, se consideran como medidas a adoptar en este ámbito:

- Optimizar las capacidades existentes: reducir gastos eliminando las obsoletas y poniendo en común las duplicadas entre estados. Esto no se puede llevar a cabo si no es bajo coordinación política de Bruselas y dirección técnica de la EDA.

- Obtención de nuevas capacidades: mediante cooperación multinacional, pero sin aspirar a incluir a todos los miembros. La CEP permite la formación de islas de cooperación por regiones que desarrollen las iniciativas de $\mathrm{P} \& \mathrm{~S}$.

- Especialización de funciones: los Estados más pequeños (sin amenazas no-compartidas) deben centrarse en cubrir carencias concretas, a cambio de la protección que garantiza la cláusula de defensa mutua del Tratado de Lisboa.

- Integración de programas: formación de grandes clúster empresariales para ganar en economía de escalas (por ejemplo, no produciendo tres cazas distintos, Eurofighter, Rafale y Grippen), e integrar el apoyo logístico y el adiestramiento.

Para todas estas medidas el marco de referencia sigue siendo el «Plan de Desarrollo de Capacidades» (CDP) de la EDA, que en la actualidad contempla como áreas prioritarias de actuación: lucha contra los artefactos explosivos improvisados, apoyo médico, medios ISR (Identification, Surveillance, Reconnaissance), helicópteros, ciberdefensa, apoyo logístico multinacional, intercambio de información PCSD, gestión del transporte aéreo estratégico y táctico, energía y combustibles, y movilidad. 
El hecho es que el desfase de capacidades con respecto a EEUU no ha dejado de crecer, comprometiendo el vínculo trasatlántico. Como afirmó el entonces secretario de defensa Gates en junio de 2011 «hay naciones que aparentemente pretenden que los contribuyentes americanos asuman la creciente carga dejada por la reducción en los prepuestos de defensa europeos», para añadir que la OTAN se divide entre los países capaces y dispuestos a pagar el precio de los compromisos y aquellos que disfrutan de los beneficios pero no quieren compartir los riesgos y los costes, algo que calificó literalmente de inaceptable ${ }^{57}$.

Dado que la UE carece de la capacidad de imponer un mínimo porcentaje de gasto a sus miembros, la única forma de progresar es el constituir una Europa de la defensa a varias velocidades. La herramienta para hacerlo es nuevamente la CEP, porque los países pueden establecer unos requisitos mínimos de participación tan exigentes como lo deseen, de modo que todo el que quiera disfrutar de sus beneficios deben esforzarse por cumplirlos. El criterio básico debería ser dedicar un porcentaje mínimo del PIB a seguridad y defensa ${ }^{58}$.

Otro tema de debate en el seno de la UE es el de los Grupos de Combate, con los países divididos entre aquellos que consideran urgente poner en práctica el concepto con su despliegue, y los que abogan por una revisión completa del concepto antes de su uso. En la reunión informal de ministros de defensa de los días 18 y 19 de febrero de 2015, celebrada en Riga, la Alta Representante Mogherini pareció abogar por la segunda opción, al afirmar que el uso potencial de los BG debería estar ligado a la identificación previa de las zonas de crisis, a lo que los representantes franceses replicaron que la UE pone en marcha constantemente nuevos conceptos sin llegar a usarlos nunca ${ }^{59}$.

El uso de los BG ha representado siempre un problema por dos razones principales: la falta de voluntad política, ya que su despliegue requiere de una decisión unánime de los Estados miembros, y el hecho de que las naciones marco que aportan las fuerzas deben igualmente correr con todos los gastos, ya que el mecanismo común de financiación «Athena» apenas aporta un $10 \%$ del presupuesto de esas operaciones. El Reino Unido se

57 The Security and Defense Agenda (Future of NATO), Bruselas, 10 de junio de 2011: http://www.defense.gov/speeches/speech.aspx?speechid=1581 (última consulta 13/03/2015).

58 En 2010 Reino Unido, Francia y Alemania sumaban 115.000 de los 194.000 millones de $€$ del total de la UE. Si a esa cifra se suman los 48.000 millones aportados por Italia, España, Países Bajos y Polonia, se concluye que los restantes 20 estados miembros gastaron 31.000 millones, de modo que todos juntos no alcanzan el presupuesto de uno solo de los «tres grandes».

59 Europe Diplomacy \& Defence, n. $^{\circ} 771$, Bruselas, Agence Europe, 21 de febrero de 2015, pp. 2-3. 
opone sistemáticamente a ampliar los fondos de «Athena»y al uso de los $\mathrm{BG}$, pero también Alemania es muy reticente a comprometerse a desplegar tropas en África, como se comprobó en el mencionado caso de EUFOR RDC.

Finalmente, hay una cuestión fundamental que ha sido identificada por todos los expertos como una de las principales carencias de la UE: la inexistencia de un Cuartel General permanente de nivel estratégico-militar. El Centro de Operaciones activado en Bruselas para coordinar las misiones en el Cuerno de África debería ser el futuro núcleo de esa nueva capacidad, y si no hay acuerdo a 28 por la oposición del Reino Unido, debería constituirse por medio de una CEP liderada por el llamado Trío de Weimar + Dos (Alemania, Francia y Polonia + Italia y España).

En conclusión, los problemas en la cooperación militar y el desarrollo de capacidades de la PCSD no radican en la falta de identificación de las medidas concretas a adoptar, sino en la carencia de voluntad para acometerlas. 